\title{
Experimental characterization of the acoustic behavior of micro-perforations under grazing flow
}

\author{
Simon Vandemaele* and Hervé Denayer ${ }^{\dagger}$ \\ KU Leuven, Leuven, Belgium \\ DMMS lab, Flanders Make, Belgium \\ Wim De Roeck \\ KU Leuven, Leuven, Belgium \\ Wim Desmet ${ }^{\S}$ \\ KU Leuven, Leuven, Belgium \\ DMMS lab, Flanders Make, Belgium
}

\begin{abstract}
By downsizing perforation dimensions to the sub-millimeter range, micro-perforated panels exhibit high enough acoustic resistance to be used without the addition of porous damping material. As a result, they can be used to design more compact acoustic wall treatments for flow duct applications. In order to fully exploit their potential, however, the effect of flow on the panel characteristics should be better understood. Within this paper, the effect of grazing flow on the acoustic impedance of sub-millimeter perforations is investigated experimentally using a multi-microphone impedance tube. Measurements are conducted for different grazing flow rates and for different inclination angles of the perforation with respect to the plate surface. It is observed that increasing the flow rate increases the resistive and decreases the reactive part of the impedance. The respective increase and decrease also seems to scale with flow rate. Next, it is detected that both the inclination angle and inclination orientation with respect to the flow direction has a significant effect on the measured impedance. Inclining the perforation against the flow direction results in a higher resistance. Furthermore, depending on the inclination orientation with respect to the flow, the frequency dependency of the reactance changes. As a consequence, the reactive curves cross at a frequency which is determined by flow rate and inclination angle.
\end{abstract}

\section{Introduction}

$\mathrm{C}$ ONVENTIONAL acoustic wall treatments for flow duct applications typically consist of a perforated facing sheet, backed by a cavity or a honeycomb structure. To reach desired levels of acoustic absorption, the cavities are often filled with additional porous or fibrous material, leading to heavy, bulky solutions. Replacing the perforated facing sheet with a thin micro-perforated panel (MPP) in which the perforations are limited to the sub-millimeter range can increase the acoustic absorption of the panel itself eliminating the need for extra porous material. As a result, more compact silencers could be constructed.

The main idea behind the principle of MPP is to downsize the perforation dimensions such that the acoustic boundary layers fill up almost the entire cross-section of the perforation. This way, the viscous forces inside the perforation are increased which results in enhanced acoustic dissipation. Furthermore, if the perforation ratio is kept low, around 1\%, the resistance of MPP approaches the characteristic impedance of air. Although this concept was originally proposed for room acoustic applications [1-3], recent studies started looking at their potential for flow duct applications as well [4-6]. By using MPP as wall treatment in a flow acoustic environment, the oscillating shear layers over the micro-perforations interact with the acoustic perturbations, affecting the acoustic characteristics of the panels and their acoustic energy dissipation. Many studies report e.g. the influence of grazing flow on the acoustic impedance of the MPP, showing that a grazing flow typically increases the resistive part and decreases the reactive component. Several attempts to capture this

\footnotetext{
*PhD. candidate, Dept. of Mechanical Engineering, Celestijnenlaan 300, 3001 Leuven, Belgium. simon.vandemaele@kuleuven.be

†Senior researcher, Dept. of Mechanical Engineering, Celestijnenlaan 300, 3001 Leuven, Belgium.

$\doteqdot$ Associate Professor, Dept. of Mechanical Engineering, Celestijnenlaan 300, 3001 Leuven, Belgium.

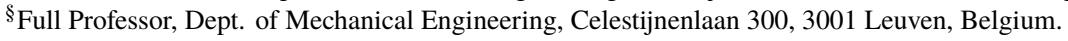


behavior quantitatively resulted, however, in different empirical correction factors, each focusing on different physical parameters $[4,7,8]$.

The detailed investigation of sub-millimeter perforations in a flow acoustic environment therefore remains necessary in order to get more insight into the physical mechanisms, the parameters influencing them and the ways to optimally exploit them. In previous work, the authors used a numerical framework based on the linearized Navier-Stokes equations to study the effect of grazing flow on sub-millimeter orifices that were inclined with respect to the flow direction. Depending on the orientation of the inclination (against or with the flow direction), a clear difference in acoustic impedance was observed [9]. In this paper, a framework is presented to verify these observations experimentally.

The experimental investigation of the acoustic properties of perforations and orifices in a grazing flow environment has received a lot of attention since the second half of the twentieth century, resulting in a wide variety of possible measurement techniques. Ronneberger [10] measured the radiation impedance of millimeter sized circular holes in the wall of flow ducts by positioning a measuring cavity below them. With this technique, the perforation impedance could be defined as the ratio of the pressures measured in the duct and cavity, but this is only valid when the particle velocity through the hole is zero. To measure the acoustic impedance of a liner sheet, Dean [11] proposed an in-situ method using two-microphones, one at the bottom of the liner cavity and one in front of the liner surface. Positioning of the latter microphone is, however, cumbersome and this microphone will not only measure acoustic, but also hydrodynamic pressure fluctuations. A more promising method is based on a grazing flow impedance tube set-up, as used by Kooijman et al. for their investigation of the flow acoustic behavior of orifices [12]. With this configuration, the impedance can be calculated from pressure measurements far away from the perforated sheet. Furthermore, these measurements take place outside of the grazing flow region, ensuring that only acoustic and no aerodynamic pressure fluctuations will be measured. Within this paper, a similar approach will be used to study the impedance of sub-millimeter perforations in a grazing flow environment.

This paper is structured as follows. The first section defines the concept of acoustic impedance and describes how to calculate this property with an impedance tube set-up. Next, the experimental set-up and methodology are described, followed by a short description of the perforated cases under consideration. Finally, the results obtained with this experimental framework are presented and discussed, leading to a short conclusion and outlook.

\section{Acoustic behavior of sub-millimeter perforations}

\section{A. Acoustic impedance of micro-perforations}

To characterize the local acoustic behavior of a perforated surface, the normalized acoustic impedance can be defined in frequency domain as:

$$
z=\frac{1}{S} z_{p}=\frac{1}{\rho_{0} c_{0}} \frac{1}{S} \frac{p_{p}}{u_{p}}
$$

In this equation, $p_{p}$ and $u_{p}$ are the acoustic pressure and normal particle velocity at the perforation opening and $\rho_{0} c_{0}$ is the characteristic impedance of the considered fluid (with $\rho_{0}$ the density and $c_{0}$ the speed of sound). $S$ denotes the surface area associated to the perforation and is the scaling factor linking the normalized impedance of the panel with the normalized impedance of a single perforation $z_{p}$. Since for perforations the acoustic wavelengths of interest are much larger than the perforated cross-section, both pressure and velocity fluctuations can be considered locally incompressible and the impedance can be calculated as an impedance jump between the two sides of the panel denoted with subscripts 'in' and 'out':

$$
\Delta z=z_{\text {in }}-z_{\text {out }}=\frac{1}{\rho_{0} c_{0}} \frac{1}{S}\left(\frac{p_{p, \text { in }}}{u_{p, \text { in }}}-\frac{p_{p, \text { out }}}{u_{p, \text { out }}}\right)=\frac{1}{S}(r+i \chi)
$$

Physically, the acoustic impedance can be interpreted by considering the real and imaginary part of this complex quantity separately as shown in the right-hand side of Eq. (2). The real part, $r$, is referred to as the resistance of the perforated sheet and accounts for the acoustic losses. As illustrated on the left of Fig. 1 for a quiescent environment, these losses are caused by visco-thermal effects in the perforation opening and on the perforation surface. The latter occur close to the perforation where the acoustic streamlines are distorted. For MPP, this resistance term is often considered to be frequency independent. The imaginary part, $\chi$, is called the reactance and represents the inertia of the air in and around the perforation as indicated with the dark blue shaded area on the left of Fig. 1. For ease of representation and calculation, Rayleigh [13] represented the occurring inertial effect as an idealized moving piston with the same air mass 


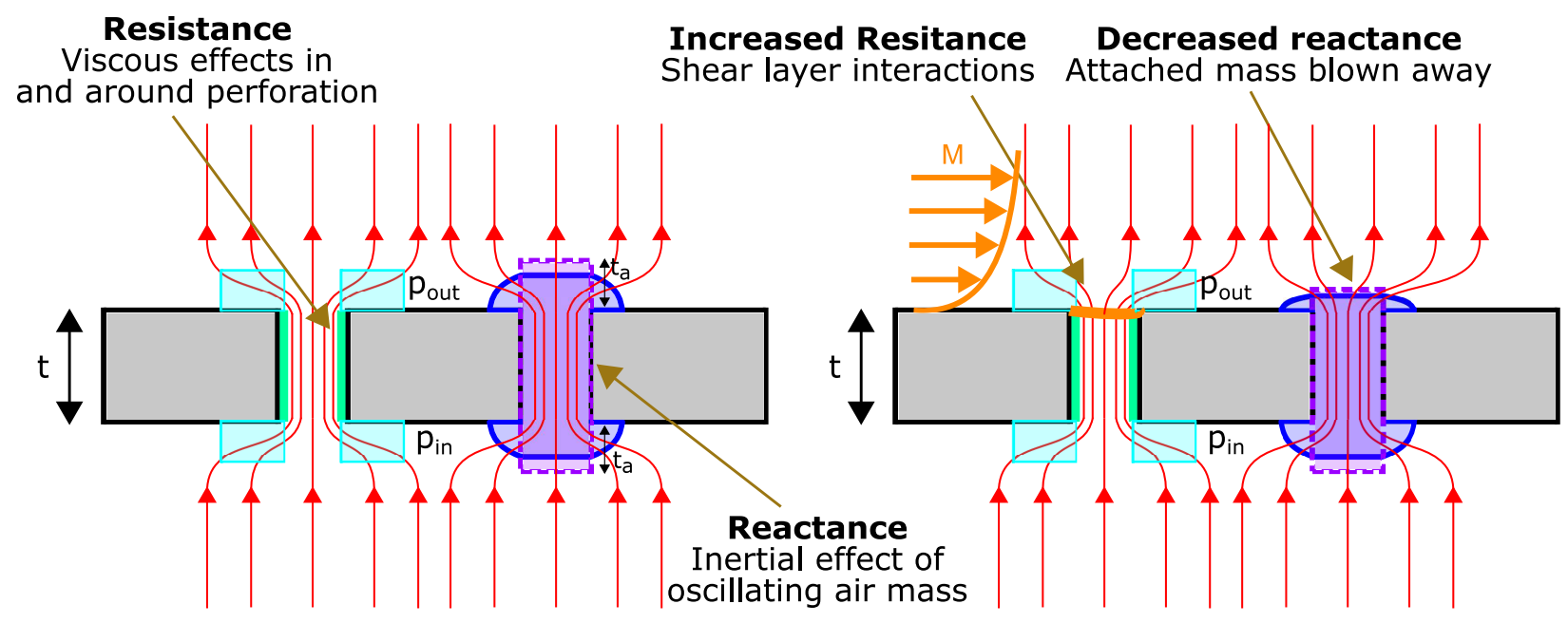

Fig. 1 (Left) Illustration of the physical meaning of the resistance and reactance term of the perforation acoustic impedance. (Right) Adding grazing flow on top of the perforation will in general increase the resistive component and decrease the reactive part.

(purple shaded rectangle on the figure). The height of the idealized air volume is hereby referred to as the effective length $t_{e}$ which is the sum of the perforation thickness $t$ and a correction term $t_{a}$, called the attached length, for the externally moving air mass. With this representation, the reactive term is proportional to the product $\chi \sim k t_{e}$ of the acoustic wavenumber $k=\frac{2 \pi f}{c_{0}}$ (with $f$ the frequency) and the effective length.

The acoustic impedance of an MPP will be altered in the presence of grazing flow, as illustrated on the right of Fig. 1. Acoustic waves interact with the created shear layer over the perforation opening leading to an energy transfer between the acoustic and aerodynamic fields which increases the perforation resistance. Furthermore, part of the oscillating air mass at the flow side is blown away which decreases the reactance [10]. Although this qualitative behavior has been reported by several studies $[4,7,8]$, it must be noted that the specific nature of these flow acoustic interactions is not yet fully understood [14].

\section{B. Measurement of the perforation impedance}

In order to effectively evaluate Eq. (2) the term $\frac{1}{\rho_{0} c_{0}} \frac{1}{S}\left(\frac{p_{p, \text { in } / \text { out }}}{u_{p, \text { in } / \text { out }}}\right)$ needs to be measured on either side of the perforation. This can be done by choosing a measurement point at each side of the perforation opening, where both pressure and velocity are measured. However, the considered measurement point cannot be located too close to the perforated surface, where the assumption of plane waves is invalid, or too far from it, making the measured impedance not representative for the perforation impedance. Moreover, in this research the effect of grazing flow (on the out side of the plate) on the acoustic perforation impedance will be investigated. Measurements in the vicinity of the perforation opening will therefore not only contain acoustic perturbations, but also aerodynamic perturbations which are created in the shear layers over the perforation openings. Splitting procedures to extract the acoustic data exist, but are rather complex [15] and are therefore avoided.

To alleviate these problems at the no flow side, an indirect measurement approach is proposed by mounting an impedance tube below the perforated sample as illustrated with the sketch in Fig. 2 [12]. Assuming plane wave propagation, the pressure at the no flow side of the MPP can be calculated based on a limited number of pressure measurements along the impedance tube length (indicated in blue).

\section{Plane wave propagation in a duct}

Below the cut-off frequency of the first cross-sectional mode of a duct (e.g. the impedance tube on Fig. 2), propagating acoustic waves can be considered plane waves and the acoustic disturbance quantities $p\left(f, z_{i}\right)$ and $u\left(f, z_{i}\right)$ 


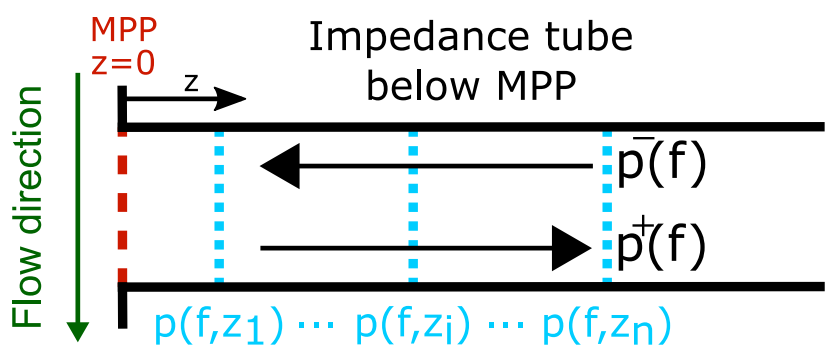

Fig. 2 Sketch of an impedance tube mounted below a perforated surface. The MPP reference position is indicated in red and the used convention of complex plane wave amplitudes $p^{ \pm}$in black. In blue, different positions are indicated to record the necessary pressure data for the plane wave decomposition procedure.

at an arbitrary position $z_{i}$ along the tube axis can be written in frequency domain as

$$
\begin{array}{r}
p\left(f, z_{i}\right)=p^{+}(f, 0) e^{-j k^{+} z_{i}}+p^{-}(f, 0) e^{j k^{-} z_{i}} \\
u\left(f, z_{i}\right)=\frac{1}{\rho_{0} c_{0}}\left(p^{+}(f, 0) e^{-j k^{+} z_{i}}-p^{-}(f, 0) e^{j k^{-} z_{i}}\right)
\end{array}
$$

In this equation, $p^{ \pm}(f, 0)$ and $k^{ \pm}$represent the complex amplitudes (at a chosen reference position $z=0$ ) and wavenumbers of the waves traveling in positive and negative z-direction. In the absence of flow in the impedance tube, these wavenumbers $k^{ \pm}$are equal and can be expressed as the acoustic wavenumber $k_{0}$ corrected for visco-thermal damping losses at the duct walls with the complex, frequency dependent, Kirchhoff coefficient $\theta(f)$ [16-18]:

$$
\left.k^{ \pm}=k_{0} \theta(f) \quad \theta(f)=1+\frac{(1-j}{\sqrt{2} S h}\right)\left(1+\frac{\gamma-1}{\sqrt{P r}}\right) \quad S h=R_{h} \sqrt{\frac{2 \pi f}{v}}
$$

with $\gamma, v$ and $\operatorname{Pr}$ the heat capacity ratio, kinematic viscosity and Prandtl number of air. The dimensionless parameter $S h$ is the shear wave number and is given by the ratio of the hydraulic radius $R_{h}$ of the considered cross-section to the oscillating viscous boundary layer thickness. The visco-thermal losses in the bulk are orders of magnitude smaller than the wall losses and can be neglected.

From Eqs. (3) and (4) it follows that the knowledge of the pressure plane wave amplitudes $p^{ \pm}$suffices to know the pressure and velocity at any position in the impedance tube. Furthermore, only the pressure spectra at two different positions $p\left(f, z_{i}\right)$ are needed to perform a plane wave decomposition operation and to calculate these unknown amplitudes. These microphone positions can be placed far enough from the perforated surface to avoid a significant hydrodynamic contribution to the recorded pressure signals. When the microphone spacing becomes a multiple of half a wavelength, however, the created system becomes undetermined and suffers from large measurement errors. To resolve this issue, more microphones $(n<2)$ at irregularly spaced positions $z_{i}$ can be installed in the impedance tube to create an overdetermined set of equations which can be solved with a least-squares approach [19]:

$$
\left\{\begin{array}{c}
p\left(f, z_{1}\right) \\
p\left(f, z_{2}\right) \\
\vdots \\
p\left(f, z_{n}\right)
\end{array}\right\}=\left[\begin{array}{cc}
e^{-j k z_{1}} & e^{j k z_{1}} \\
e^{-j k z_{2}} & e^{j k z_{2}} \\
\vdots & \vdots \\
e^{-j k z_{n}} & e^{j k z_{n}}
\end{array}\right]\left\{\begin{array}{l}
p^{+}(f, 0) \\
p^{-}(f, 0)
\end{array}\right\}
$$

\section{Calculation of the perforation impedance}

For the considered measurement configuration, the impedance $z_{\text {out }}=\frac{1}{\rho_{0} c_{0}} \frac{1}{S}\left(\frac{p_{p, \text { out }}}{u_{p, \text { out }}}\right)$ from Eq. (2) is equal to the radiation impedance from the perforation opening inside the grazing medium at its upper side. In case the mean flow velocity is low $(M<0.2)$, the radiation impedance can be considered independent of the flow velocity as postulated by Ingard and Singhal [20]. For this reason, only the one-sided perforated surface impedance $z_{\text {in }}$ will be studied.

With the assumption of the locally incompressible flow around the perforations, the particle velocity at the perforation opening $u_{p, i n}$ is calculated by dividing the acoustic velocity in the tube $u(0)$ with $S$. Together with the expressions (3) 
and (4) and the convention introduced in Fig. 2 the impedance $z_{\text {in }}$ can be written as:

$$
z_{i n}=\frac{1}{\rho_{0} c_{0}} \frac{1}{S} \frac{p_{p, i n}}{u_{p, i n}}=-\frac{p^{+}(f, 0)+p^{-}(f, 0)}{p^{+}(f, 0)-p^{-}(f, 0)}
$$

\section{Linearity of the perforation impedance}

The behavior of micro-perforated plates can be considered linear at sufficiently low sound pressure levels. In this regime, the presented (flow)acoustic impedance will vary with changing perforation geometry or mean flow rate, but will be independent of the acoustic excitation level. Increasing the incident sound pressure level, however, will cause the acoustic particle velocity in the perforation to become so high that an acoustic jet will form at the perforation exit from which vortices can be shed [21, 22]. This process is non-linear and results in an increasing resistive and decreasing reactive component. In this work, the focus lies on the linear behavior of MPP and care is taken to keep the acoustic particle velocity in the perforation opening low enough so that non-linear effects on the impedance are kept to a minimum.

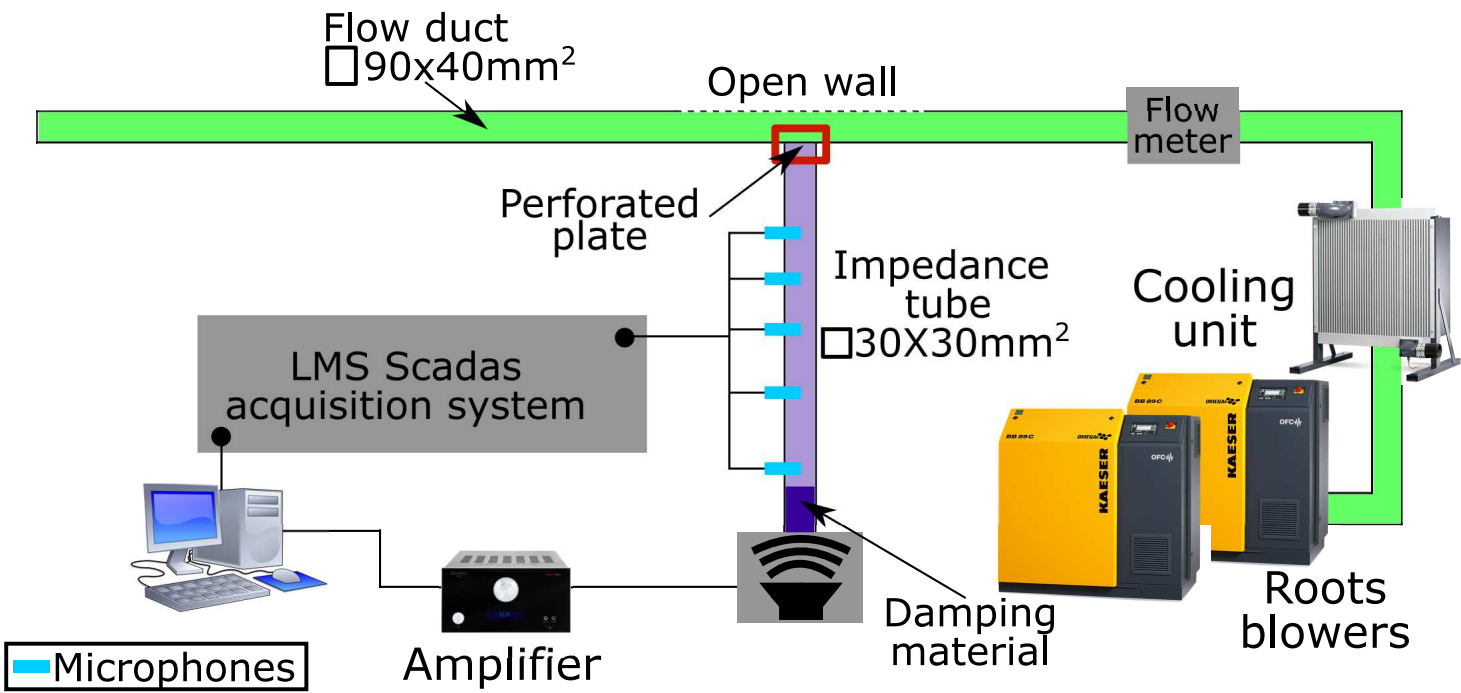

Fig. 3 Schematic overview of the experimental test set-up consisting of a main flow duct (green) and an impedance tube (purple). The perforated plate sample is positioned in between (red). Two roots blowers regulate the airflow through the duct network and a cooling unit ensures stable measurement conditions.

\section{Experimental characterization of the acoustic impedance}

\section{A. Measurement set-up}

The measurements are carried out with the flow acoustic test facilities of KU Leuven. The experimental set-up is schematically drawn in Fig. 3 and consists of a main duct network (indicated in green), a grazing flow impedance tube (indicated in purple) and the mounted test sample in between both components (indicated with the red rectangle). Two serial roots blowers (frequency regulated) regulate the airflow through the main duct which has a rectangular cross-section of $90 \mathrm{~mm}$ by $40 \mathrm{~mm}$. With this cross-section, the average Mach number can be regulated in a range between 0 and 0.2. A cooling unit is installed upstream to ensure stable measurement conditions and both the temperature and the flow rate of the air are monitored with a built-in vortex flowmeter.

At the MPP sample, the flow duct wall opposite the sample has been removed from the set-up as illustrated on the left of Fig. 4. This has been done to exclude unwanted flow acoustic interaction phenomena between the flow duct and the impedance tube that could pollute the measured impedance values. As such, the effective flow rate above the MPP sample will be slightly lower than the upstream measured value and the occurring flow profile at the perforations will slightly differ from the fully developed duct profile the test rig ensures when it would be closed. The loss in flow rate is, 

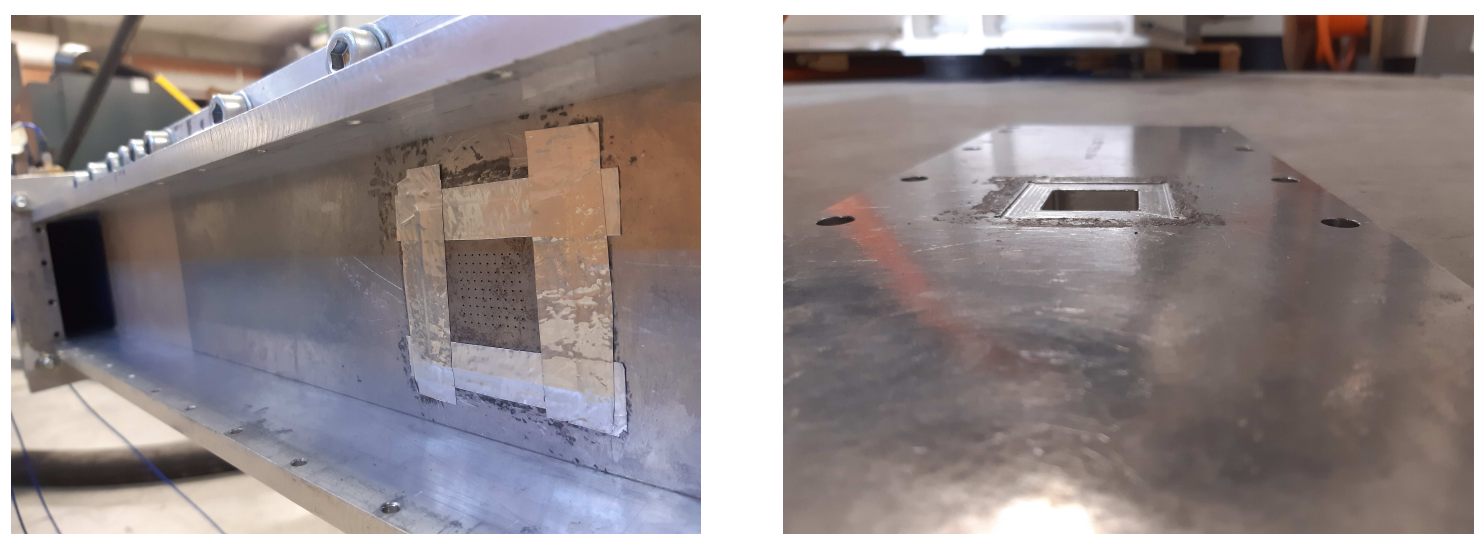

Fig. 4 (Left) Illustration of the open flow duct configuration to create grazing flow over the perforation. The perforated sample is mounted flush with the duct wall and its edges are sealed with aluminum tape. (Right) Detailed view of the flow duct sidewall in which the plate samples are mounted.

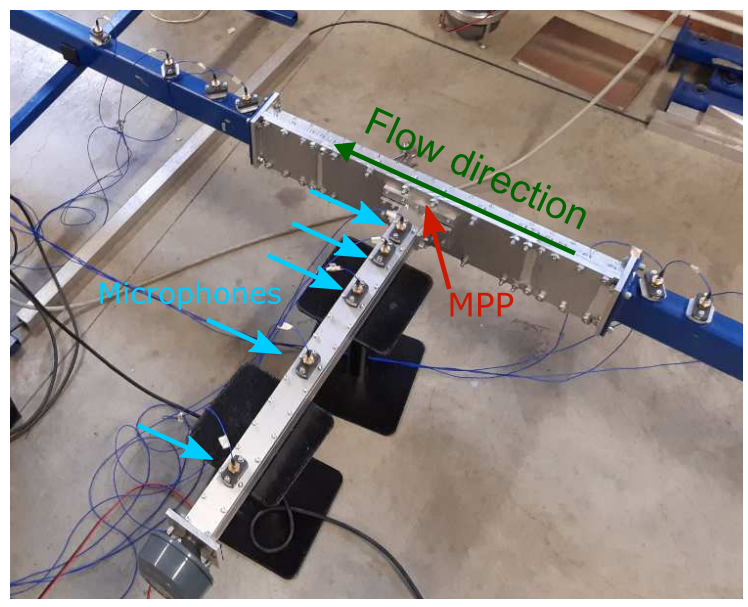

Fig. 5 Detailed view of the grazing flow impedance tube mounted into the flow acoustic test rig of KU Leuven. The MPP reference position is indicated in red and the flow direction in green. In blue, the five microphones are indicated which record the necessary pressure data for the plane wave decomposition procedure.

however, minimal and deemed acceptable for the current investigation. The determination of the specific flow profile is outside the scope of this paper.

The impedance tube is positioned perpendicular to the side wall of the main flow duct and is equipped with five flush-mounted microphones, type PCB 378C10. Figure 5 shows the practical realization of the impedance tube, which has a square cross-section with inner dimensions of $30 \mathrm{~mm}$ by $30 \mathrm{~mm}$. The first cut-off frequency for this cross-section lies around $5700 \mathrm{~Hz}$. The five microphones are irregularly spaced between $89.5 \mathrm{~mm}$ and $659.5 \mathrm{~mm}$ to make sure that, for each frequency, no more than one microphone is spaced half a wavelength from another microphone. The position of the first microphone is chosen far enough from the perforations to limit near field effects polluting the recorded pressure signals.

The aluminum walls of the impedance tube have a thickness of $10 \mathrm{~mm}$ which should be enough to limit structural vibrations and some acoustic damping material is added at the tube's extremity, in front of the loudspeaker to avoid strong standing wave resonances in the tube. An LMS Scadas system together with the Test.Lab v17 software is used for both the acquisition of the pressure data and for the generation of the excitation signal.

Figure 4 shows on the left the MPP sample mounted into the flow duct wall. The perforated sample is mounted flush with the wall and its edges are sealed with aluminum tape to minimize leakage and flow disturbances near the perforations. The right-side of the figure shows how the sample is mounted: on the inner side of the duct wall, a square cavity with the dimensions of the plate samples is milled. In this cavity, the sample is attached with double-sided tape. 


\section{B. Measurement procedure}

By definition, the concept of MPP shows a lot of potential in the lower frequency range. In this range, the resistive effect of the perforations will dominate over the reactive part due to the increasing acoustic boundary layer thickness with decreasing frequency. For this reason, the frequency range of interest is set from $150 \mathrm{~Hz}$ to $1850 \mathrm{~Hz}$, which is far below the first cut-off frequency of the impedance tube. The assumption of plane wave propagation is thus justified.

From Eq. (6) it follows that the calculation of the pressure wave amplitudes $p^{ \pm}$solely depend on the plane wave decomposition. By using more than two microphones, the influence of measurement errors is already reduced by overdetermination of the system. However, to further improve the accuracy of this step, additional measures are taken. First, instead of using the measured pressure fluctuations $p\left(f, z_{i}\right)$ directly, they are replaced with the transfer functions $H\left(f, z_{i}\right)$ between the measured pressure fluctuations and a clean reference signal, in this case the excitation signal sent to the amplifier. With this technique, uncorrelated pressure fluctuations can be discarded from the measurements. Second, an appropriate, excitation signal is selected, together with a suitable number of spectral averages. For the no flow cases, a random excitation signal with 100 averages is used, whereas a stepped sine approach $(50 \mathrm{~Hz}$ frequency step, 15 averages) is selected when turbulent flow is present over the perforations. Third, the excitation level is set sufficiently high to ensure a proper signal-to-noise-ratio. Fourth, the use of multiple microphones allows the use of the iterative procedure from reference [23], which solves the overdetermined set of equations (Eq. (6)) not only for the unknown pressure wave amplitudes, but also for additional environmental parameters such as the speed of sound $c_{0}$ to reduce the uncertainties on the result.

As stated before, perforated plates are known to exhibit non-linear behavior when the acoustic particle velocity in the perforation becomes too high. To judge whether or not a non-linear acoustic response is observed, measurements are carried out with different amplifier settings.

\section{Investigated micro-perforated plate samples}

The measured test samples are depicted in Fig. 6 . The square steel plates have a side of $50 \mathrm{~mm}$, a thickness of $0.5 \mathrm{~mm}$ and contain 81 perforations with a diameter of $0.5 \mathrm{~mm}$. Using an electrical discharge machining method, the perforations are equidistantly drilled in a square pattern with a centerline-to-centerline distance of $2.5 \mathrm{~mm}$. This value corresponds to 5 times the perforation diameter and matches the theoretical limit postulated by Melling at which interaction effects between neighboring perforations can be neglected [24]. Calculated with the inner cross-section of the impedance tube, the perforation ratio of the samples is $1.77 \%$. To investigate the effect of perforation inclination angle, perforations were drilled under $0^{\circ}, 15^{\circ}, 30^{\circ}, 45^{\circ}$ and $60^{\circ}$. Figure 7 defines the inclination angle $\theta$ as the angle between the drilling axis and the direction normal to the plate. Positive angles refer to an inclination against the flow direction. It is important to notice that inclining the perforation increases both the length of the perforation $t_{i}$ and the perforation ratio as can be seen on the figure. Both parameters change as follows:

$$
S_{i}=\frac{S}{\cos (\theta)} \quad t_{i}=\frac{t}{\cos (\theta)}
$$

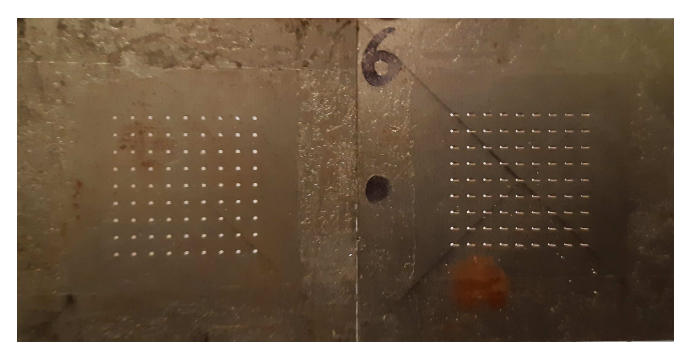

Fig. 6 Overview of two perforated plate samples with a different inclination angle: $0^{\circ}$ (left) and $60^{\circ}$ (right). The perforations are made with electrical discharge machining.

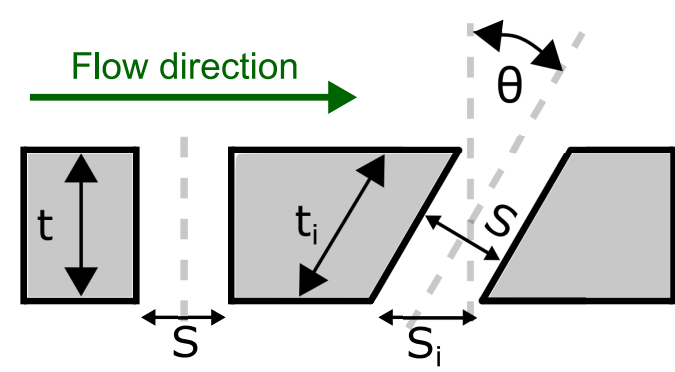

Fig. 7 Illustration of the inclination angle $\theta$ and the inclination orientation 'against the flow'. Inclining the perforation increases its length $t_{i}$ and its perforation ratio. 


\section{Discussion of the results}

\section{A. Acoustic impedance without grazing flow}

At first, the impedance of the perforated samples is measured in quiescent conditions. To identify the linear regime of the perforated samples, measurements at different acoustic excitation levels were carried out for the not inclined perforation sample. Figure 8 defines for three frequencies the dependency of the resistance (left) and reactance (right) component with the particle velocity through the orifices which can be calculated from Eq. (4) scaled with the perforation ratio. A linear response is detected at sufficiently low particle velocity defined by the constant resistance and reactance. Increasing the particle velocity through the perforations leads to an increasing resistance and decreasing reactance as described in literature [21, 22]. Based on this figure, the excitation levels used to determine the proceeding impedance results were adapted to stay in the linear regime.

The impedance results in a quiescent medium are shown in Fig. 9 for different inclination angles: $0^{\circ}$ (red), $15^{\circ}$ (blue), $30^{\circ}$ (green), $45^{\circ}$ (purple) and $60^{\circ}$ (orange). The results are scaled with the correction factor from Eq. (8) to account for the increased perforation ratio with inclination angle. The real part of the impedance, the resistance, is plotted on the left graph, the imaginary part, the reactance, on the right. At the lower frequencies, the resistive part slightly increases with frequency. From $900 \mathrm{~Hz}$ onward, however, a faster increase is detected. Measuring at low frequencies is challenging due to the limited length of the impedance tube, whereas measuring at higher frequencies could be influenced by the structural dynamics of the thin plate. At this point in the investigation, it therefore still needs to be validated whether this change in resistive behavior is a pure acoustic feature from the perforations or whether it is caused by the specific measurement set-up. As expected, a clear linear dependency with frequency is observed for the reactive component [3].

Increasing the inclination angle, clearly increases the resistance and reactance. Since the results are scaled with the perforation ratio correction factor, this increase can be attributed to the increased length of the perforations which creates a larger region of viscous scrubbing losses (higher resistance) and a larger oscillating air mass in the perforation (higher reactance). The smallest inclination angle of $15^{\circ}$ is, however, an exception to this trend showcasing a lower resistance than the $0^{\circ}$ sample at lower frequencies and a lower reactance at higher frequencies. Since this inclination angle only slightly increases the perforation length (the correction factor of Eq. (8) is 1.035), the difference with the non-inclined case was expected to be very small and could fall within the measurement uncertainty intervals. An important variable to take into account here is the plate mounting strategy, especially if the structural dynamics of the plate would play a non-negligible role. A detailed variability and repeatability study, which is outside of the scope of this paper, could yield further knowledge on the occurring phenomena.
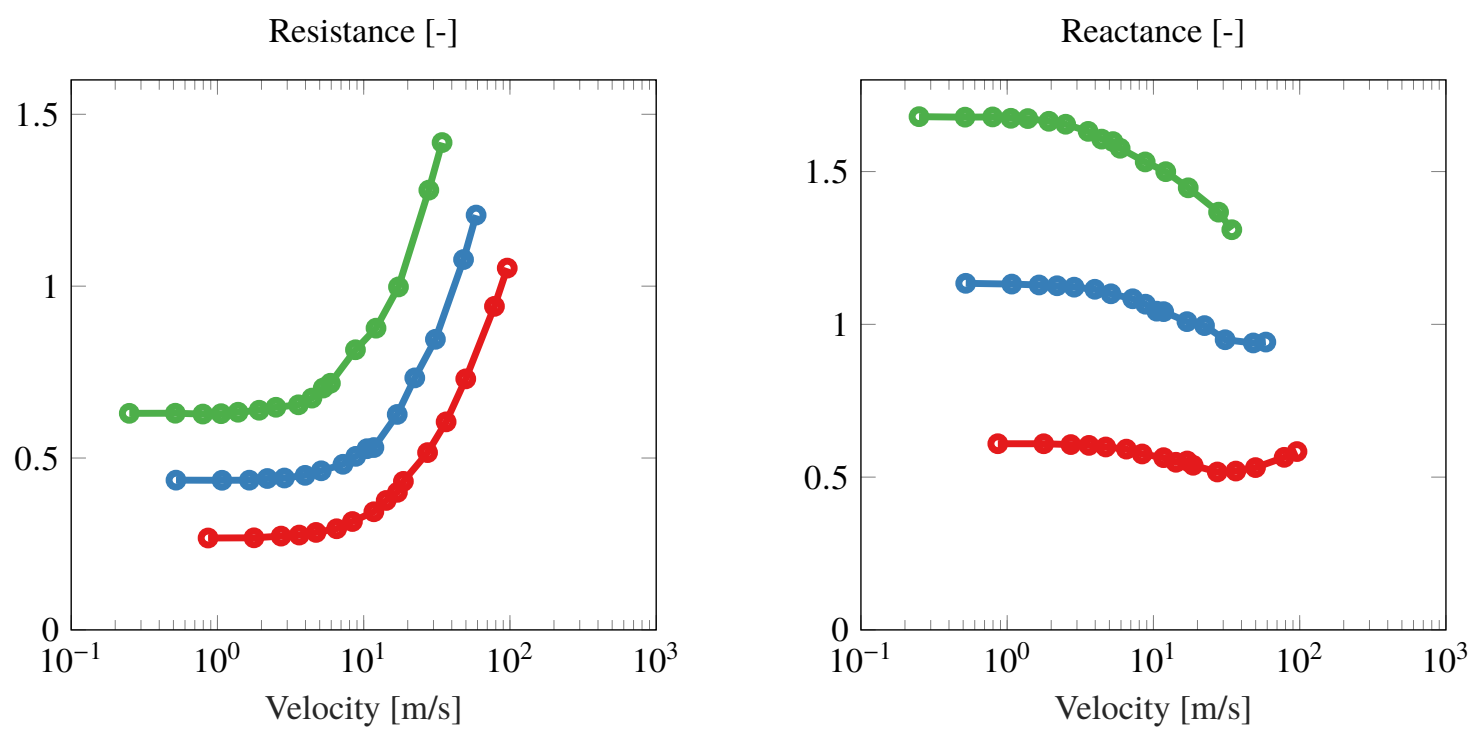

$500 \mathrm{~Hz} \rightarrow-1000 \mathrm{~Hz} \rightarrow-1500 \mathrm{~Hz}$

Fig. 8 Resistance (left) and reactance (right) dependency on the particle velocity through the perforations for $M=\mathbf{0}$ and $\boldsymbol{\theta}=\mathbf{0}^{\circ}$. 


\section{Scaled resistance [-]}

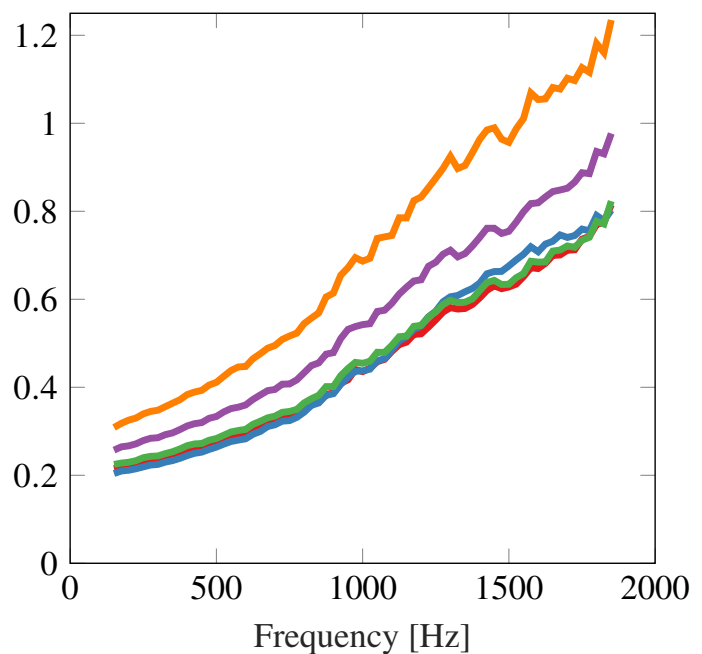

Scaled reactance $[-]$

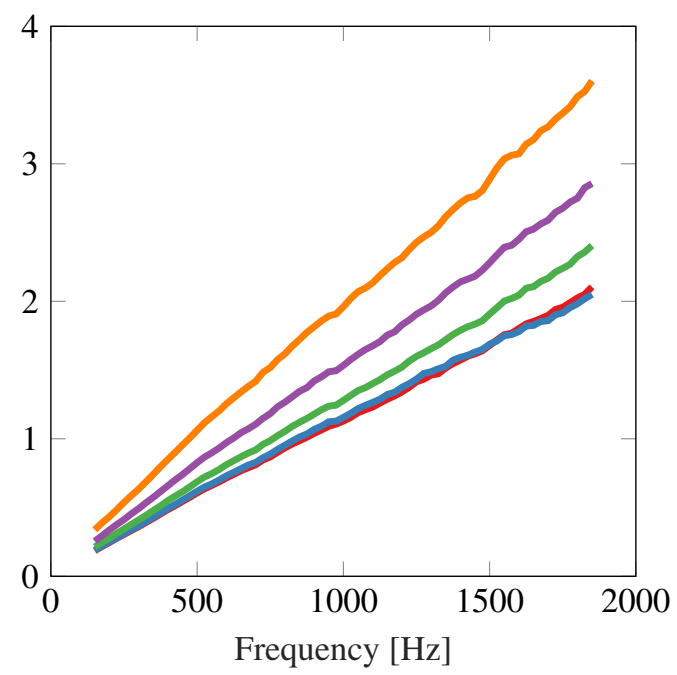

$$
\square \theta=0^{\circ}-\theta=15^{\circ}-\theta=30^{\circ}-\theta=45^{\circ}-\theta=60^{\circ}
$$

Fig. 9 Quiescent impedance of micro-perforations with an inclination angle of $0^{\circ}$ (red), $15^{\circ}(\mathrm{blue}), 30^{\circ}$ (green), $45^{\circ}$ (purple) and $60^{\circ}$ (orange). The resistive part is displayed on the left, the reactive part on the right. Both are scaled with the the factor of Eq. (8) to account for the difference in perforation ratio.

Resistance [-]

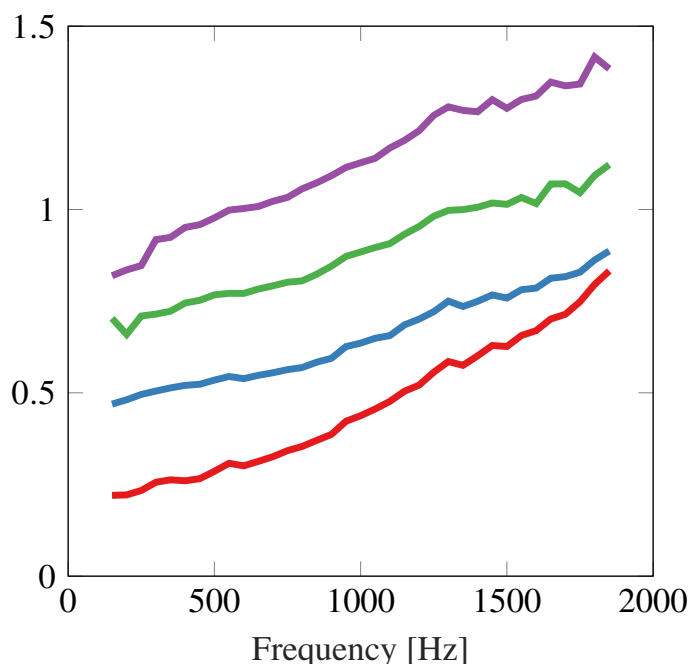

Reactance [-]

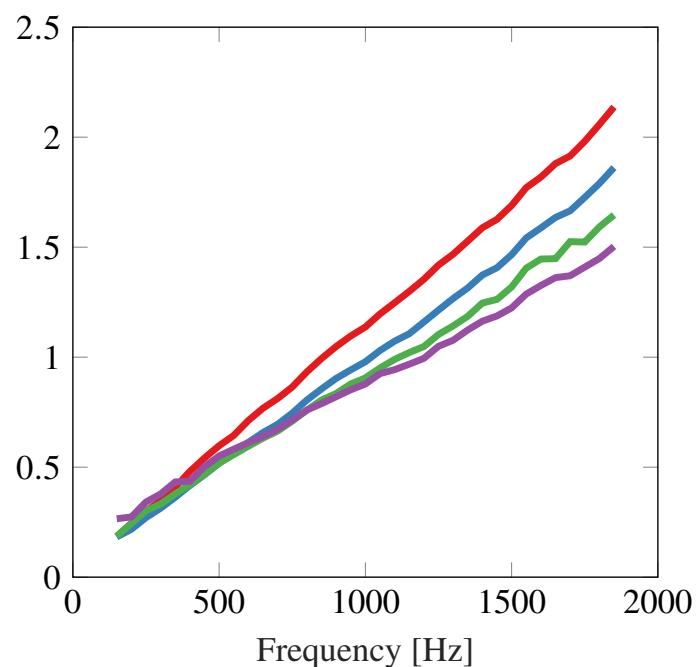

$$
\square M=0-M=0.05-M=0.075-M=0.1
$$

Fig. 10 Influence of a one-sided grazing flow ( $M=0$ (red), $M=0.05$ (blue), $M=0.075$ (green) and $M=0.1$ (purple)) on the acoustic impedance of not inclined sub-millimeter perforations: resistance (left) and reactance (right).

\section{B. Acoustic impedance with grazing flow}

The effect of a one-sided grazing flow on the acoustic impedance of the circular, not inclined, perforations is shown in Fig. 10. Three different mean flow Mach numbers are considered: $M=0.05$ (blue), $M=0.075$ (green) and $M=0.1$ (purple). The grazing flow over the perforations significantly increases the resistive component in comparison to the quiescent case (red curve), which is as expected. Furthermore, the increase scales linearly with Mach number. This trend qualitatively follows typical, Mach number based, empirical correction terms reported in literature [4, 7, 25]. 
With respect to the resistance in a quiescent medium, however, the expected parallel shift is not observed. Instead, the resistance in a moving medium seems to increase less with frequency resulting in a no flow resistance which is almost as high as the resistance for $M=0.05$ at the highest considered frequency.

On the reactive side, the expected decrease with respect to the quiescent situation is observed $[4,7,26]$, which can be explained by a reduced attached mass on top of the perforations when it interacts with the flow [10]. Like the resistance, the decreasing slope of the reactance seems to scale with the Mach number, however, this time also with respect to the no flow reactance. At lower frequencies, a slight increase is observed for $M=0.1$. However, this observation needs to be interpreted with care. Increasing the flow rate, reduces the signal-to-noise ratio and the lower frequencies are less well excited. Consequently, these more difficult measurement conditions yield a higher uncertainty.

Figure 11 depicts the influence of the inclination orientation of the perforations on the measured flow acoustic impedance for two different inclination angles: $30^{\circ}$ (top) and $60^{\circ}$ (bottom). The solid lines indicate an inclination oriented against the flow direction following the convention from Fig. 7, whereas the dashed lines are used to represent an orientation with the flow direction. For each flow rate, both orientations were measured under similar conditions. To ensure this, the sample was not removed from the sample holder, shown in Fig. 4, but instead the entire sample holder was rotated. The flow rate and temperature were not altered in this process. The considered flow rates are $M=0.05$ (red), $M=0.075$ (blue) and $M=0.1$ (green). Looking at each combination of inclination angle and orientation, the same qualitative behavior of Fig. 10 is observed at higher flow rates: a rising resistance and a lowering reactance.

Changing the inclination orientation with respect to the flow direction induces a difference in the measured
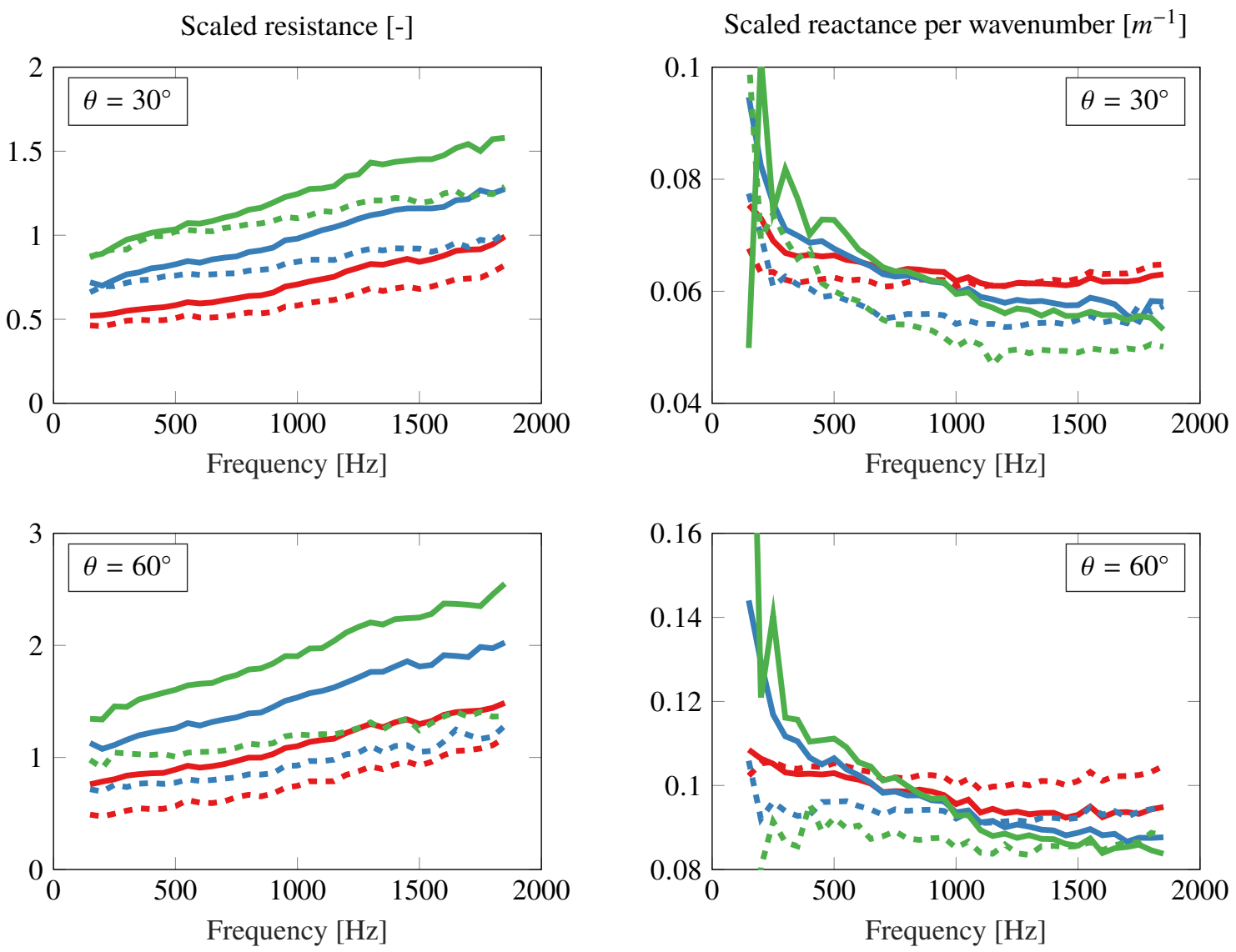

$M=0.05-M=0.075-M=0.1$
against flow direction $=-=$ in flow direction

Fig. 11 Influence of the orientation inclination on the flow acoustic resistance (left) and scaled reactance with wavenumber (right) of sub-millimeter perforations for $\theta=30^{\circ}$ (top) and $\theta=60^{\circ}$ (bottom). 
perforation resistance (left side of Fig. 11). Significantly higher resistive values are obtained when the orifices are inclined against the flow direction (solid versus dashed lines), which is in agreement to the observation made by the authors in a previously conducted numerical study on the impedance of a single square orifice under $30^{\circ}$ and $60^{\circ}$ [9]. In this paper, the authors linked the change in resistance to the occurring mean velocity profile in and around the perforation opening. Although the presence of a small bias flow component through the orifices in that numerical study is making a one-to-one comparison difficult, a similar reasoning could be made for the purely grazing flow cases of this experimental study. Adding grazing flow induces a shear layer over the orifices together with a small circulation component in the perforated opening. Inclining the perforation slightly alters the strength and shape of this shear layer and circulation component. Stronger, more sharp shear layer profiles cause a stronger "barrier" for the impinging acoustics leading to more (flow)acoustic losses and a higher resistance. To validate this theory and to specifically determine the physical phenomena at stake, follow-up research on the flow profile, both numerical and experimental, is needed.

With increasing flow rate, a change in frequency dependency of the resistance curve can be detected, especially for the $60^{\circ}$ case. At the lowest Mach number (red), the difference in resistance between both orientations seems to be frequency independent, resulting in a near parallel shift between both curves. Going to higher flow rates (blue, green), however, a larger resistance gap is detected at higher frequencies. This is caused by a change in frequency dependency of the resistance of the perforation inclined with the flow, which exhibits a less strong frequency dependency. The specific cause for this phenomenon is still under investigation.

Changing the inclination orientation also induces a difference in the measured reactance (right side of Fig. 11). To visualize and interpret the occurring changes, the scaled reactive component has been divided by the wavenumber. Since the reactance is dominated by the inertia of the oscillating air mass, division by the wavenumber is expected to result in a constant value. Especially at higher frequencies, this is observed to be true. Looking at the $30^{\circ}$ angle, a difference in both value and slope of the curves is detected. The end correction of the inclination against the flow direction is higher at low frequencies, but decreases faster with frequency. Inclining the perforation with the flow direction leads to an overall less frequency dependent behavior. As a result both curves cross. For the lower flow rate (red), this happens around $1200 \mathrm{~Hz}$, for the other flow rates this is believed to happen outside of the studied frequency range. This trend becomes more pronounced for the larger $60^{\circ}$ angle, where the crossing points shift to lower frequencies. Also for the higher flow rates, they are now detected in the frequency range of interest. This particular behavior was partly observed in the preceding numerical study carried out by the authors [9]: orienting the perforation inclination in the flow direction resulted in higher reactive values. In that paper, the crossing point of both orientation directions appeared, however, at the very low frequencies and no clear dependency with angle was detected. Although a one-to-one comparison with those numerical results is difficult due to square perforation cross-section and the presence of a small bias flow component, it seems that small alterations of the flow field in the near vicinity of the orifices can largely affect the measured impedance and its frequency dependency.

Figure 12 compares the scaled impedance values of the different inclination angles per measured flow rate: $M=0.05$ (top), $M=0.075$ (middle) and $M=0.1$ (bottom). Resistance is again depicted on the left, reactance on the right and the results of two extra angles $\left(15^{\circ}\right.$ and $\left.45^{\circ}\right)$ are added as well. First a significant larger resistance difference for larger inclination angles is detected, following the observations made in Figs. 9 and 11. This is the case for all flow rates. However, inclining the perforation angle with the flow direction minimizes this resistance increase (bottom left of Fig. 12 where the dashed curves all start to overlap). The difference for the orientation against the flow direction stays significant. As a consequence, the resistance gap between both inclination orientations increases with inclination angle and with flow rate which is illustrated by the difference $\Delta_{A-W}$ in Fig. 13 where the results of the inclination with flow direction were subtracted from the opposite inclination orientation. This plot also clearly shows the frequency dependency of the resistance difference: for higher frequencies the observed difference between both inclination orientations gets larger.

Furthermore, it is noted that the measured resistance difference is also significant for the smallest angle of $15^{\circ}$, shown in red. As depicted in Fig. 9, the quiescent impedance of this plate did not significantly differ from the non-inclined situation since the increase in perforation length is limited. The $15^{\circ}$ case showed even slightly higher resistance at higher frequencies compared to the $30^{\circ}$ angle configuration. This effect can still be seen in Fig. 12 for the inclination aligned with the flow direction (e.g. dashed red and blue lines on the top left), but it is absent for the opposite orientation. As a result, the resistance difference shown in Fig. 13 for the $30^{\circ}$ angle is significantly higher than the result for $15^{\circ}$.

The crossing point of the measured reactance curves of both orientations can be spotted on the right side of Fig. 12 . In the plot of Fig. 13, this crossing frequency is represented as the point where the reactive difference becomes zero. Here it is seen that for the angles $15^{\circ}, 30^{\circ}$ and $45^{\circ}$ the observed reactive difference follows a similar trend and the crossing frequency seems to coincide. However, the used frequency resolution of $50 \mathrm{~Hz}$ could mask an occurring frequency shift so no hard statements can be made. For the largest angle of $60^{\circ}$ the crossing frequency shifts to lower 
Scaled resistance $[-]$
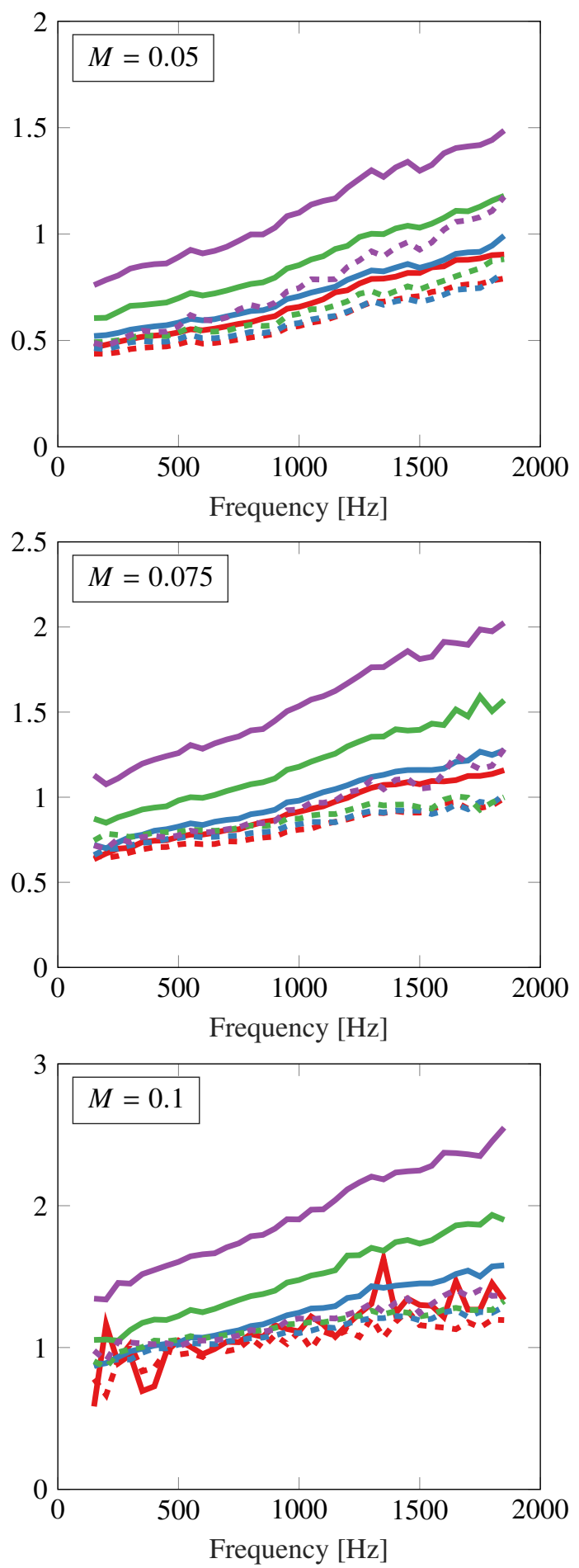

Scaled reactance [-]
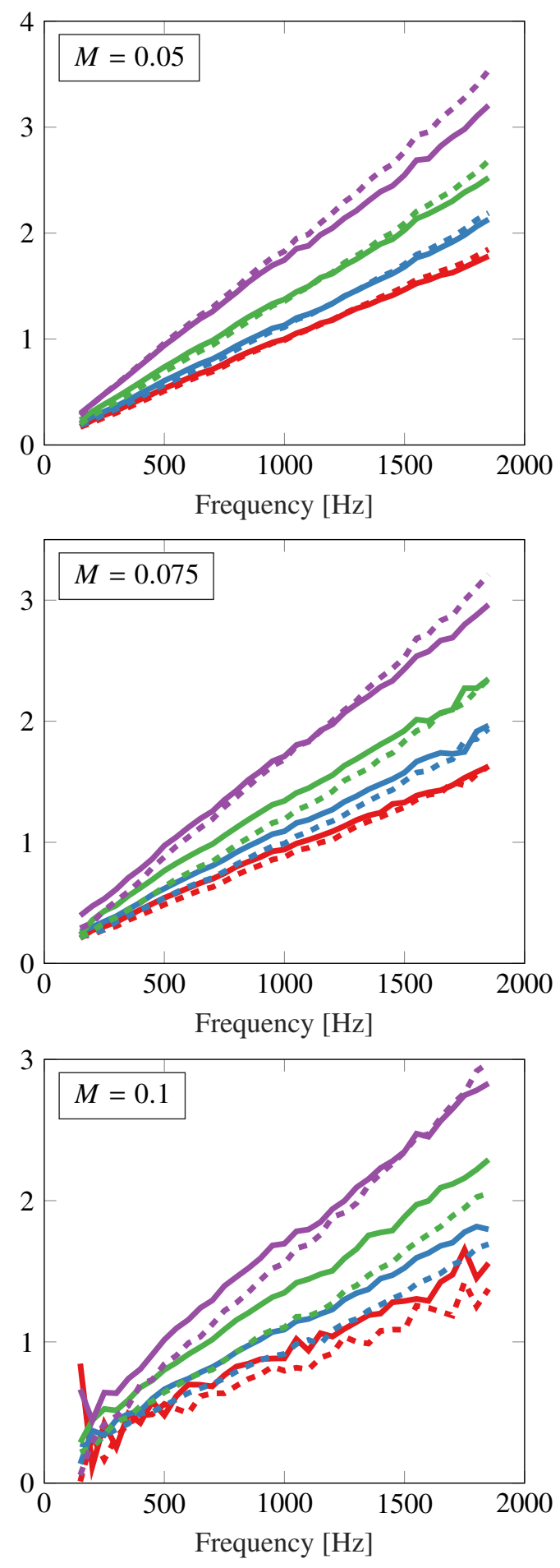

$-\theta=15^{\circ}-\theta=30^{\circ}-\theta=45^{\circ}-\theta=60^{\circ}$

against flow direction $==-$ in flow direction

Fig. 12 Influence of the orientation inclination on the flow acoustic resistance (left) and reactance (right) of sub-millimeter perforations for $M=0.05$ (top), $M=0.075$ (middle) and $M=0.1$ (bottom). The results are shown for different inclination angles. 
$\Delta_{A-W}$ scaled resistance [-]
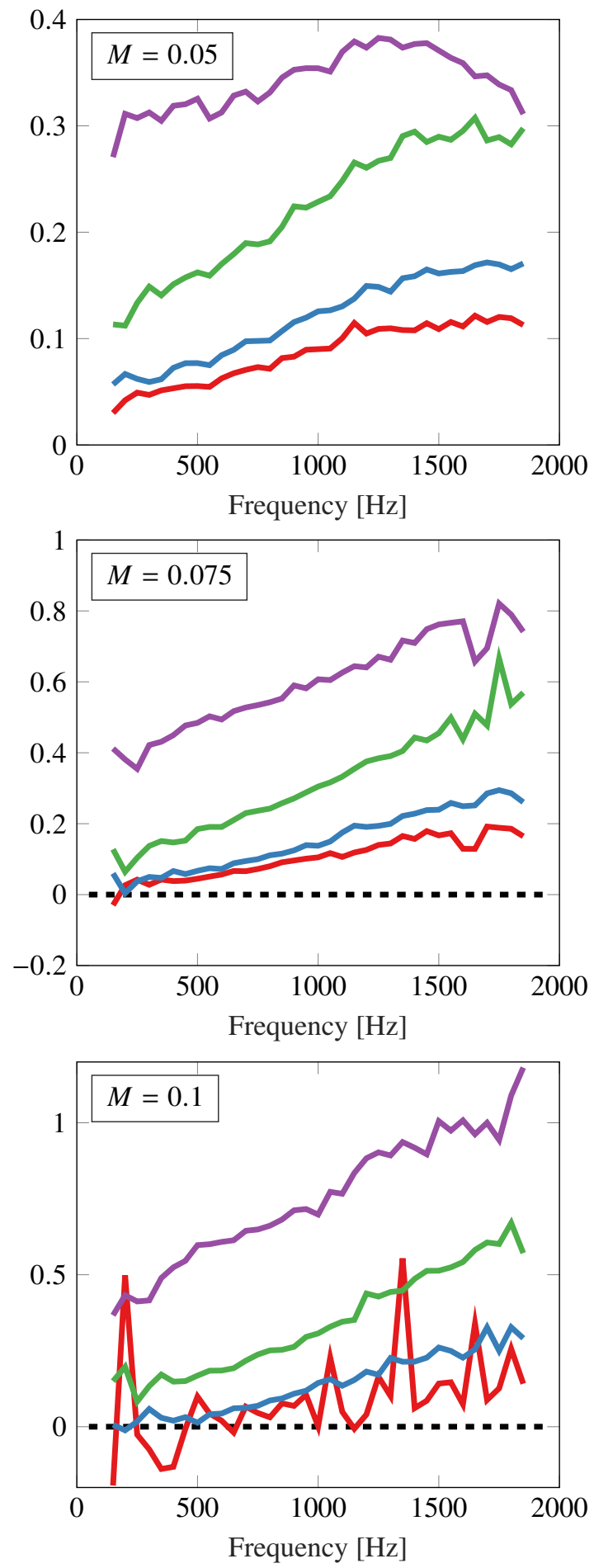

$\Delta_{A-W}$ scaled reactance [-]
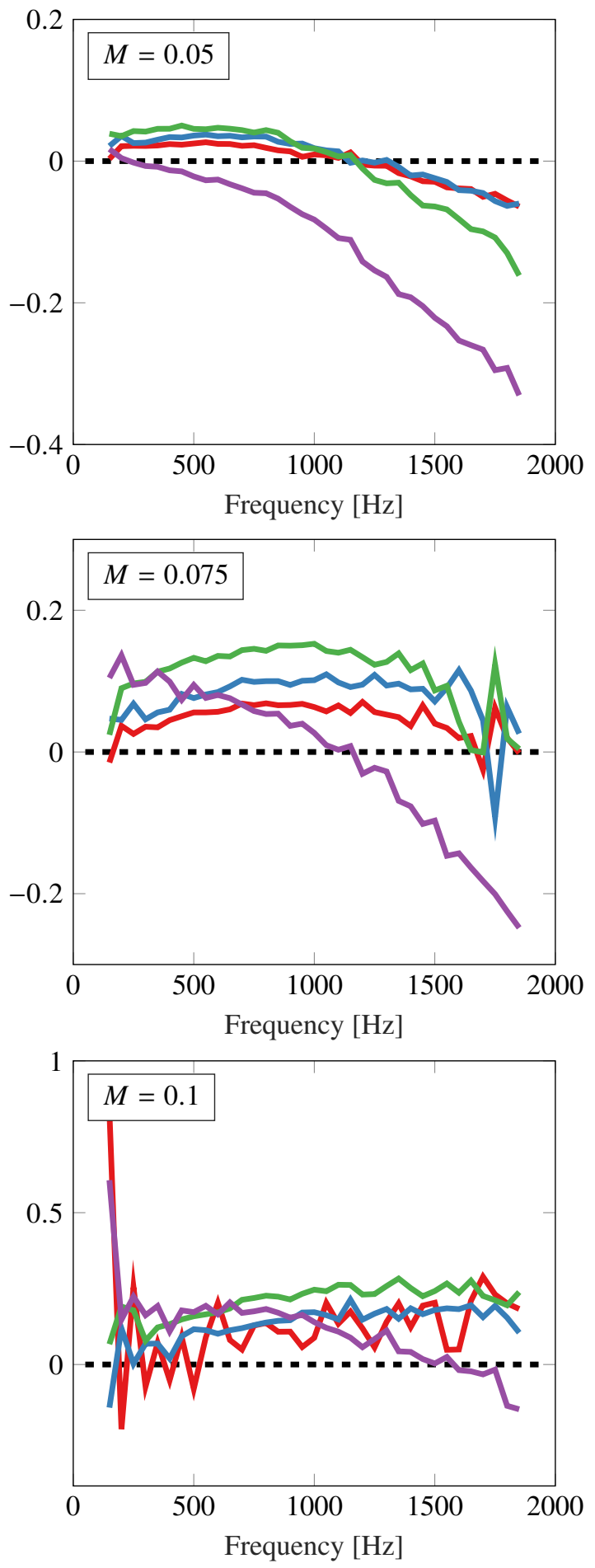

$$
\square \theta=15^{\circ}-\theta=30^{\circ}-\theta=45^{\circ}-\theta=60^{\circ}
$$

Fig. 13 Measured resistive (left) and reactive (right) difference $\Delta_{A-W}$ between both inclination directions for different angles and flow rates. The difference is calculated by subtracting the results for an inclination in flow direction (dashed lines from Fig. 12) from those obtained with an inclination against flow direction (solid lines from Fig. 12). 
frequencies. As stated before, increasing the flow rate, increases this frequency and for the highest flow rates and smaller angles (red, blue and green on the bottom right graph), this point does not appear in the studied frequency range.

Finally, it is observed that the measurement of the $15^{\circ}$ angle for $M=0.1$ shows some irregular deviations from the expected smooth behavior obtained for the other angles. Since measuring at the higher flow rates adversely affects the signal-to-noise ratio, this could be due to an accumulation of measurement uncertainties rather than some flow acoustic phenomenon. The observed discrepancies in the quiescent impedance for this flow rate (Fig. 9) supports this hypothesis, but further validation is needed.

From these results it is clear that a change in inclination orientation leads to significant changes of the perforation impedance. These changes are believed to be attributed to the altered flow field and shear layer strength in and around the perforation opening. The specific nature and relevant parameters of this interaction are, however, still under investigation.

\section{Conclusions and future work}

This paper presents a grazing flow impedance tube experimental test rig to investigate the flow acoustic behavior of micro-perforations. The perforated samples have a perforation diameter and plate thickness of $0.5 \mathrm{~mm}$ and are mounted in the sidewall of a flow duct, subjected by a varying grazing flow between Mach 0 and 0.1 . Based on pressure measurements in an impedance tube, positioned at the lower side of the plate, the one-sided acoustic impedance is calculated and the influence of the flow on both the resistive (real) and reactive (imaginary) component is assessed. Care is taken to measure the impedance in the linear regime by monitoring the particle velocity through the orifices.

In quiescent conditions the reactive component scales linearly with frequency as expected. The resistive part slightly increases at lower frequencies, but starts to increase faster at higher frequencies. The latter is peculiar and it is still under investigation if unwanted effects like the dynamics of the thin plate have a non-negligible influence on the results. Inclining the perforation with respect to the plate surface increases both the perforated area and the perforation length. The latter results in higher resistance and reactance values which is illustrated by considering inclination angles ranging from $0^{\circ}$ to $60^{\circ}$.

In a grazing flow environment a shear layer is formed over the perforation opening which typically reduces the effective length of the perforations. As a result the reactance decreases. Furthermore, acoustic-aerodynamic interactions occur, transferring energy from the acoustic to the aerodynamic field, which increases the resistance. Both trends are detected in the measured impedance values and it seems that the decrease/increase scales with the flow rate.

Measurements performed on the inclined perforation cases reveal a clear dependency of the impedance in a moving medium on the inclination orientation. Inclining the perforation against the flow direction (center-line of the perforation points downstream) results in higher resistance values compared to an inclination with the flow direction. For higher flow rates, the observed resistance difference seems to slightly increase at higher frequencies. Inclining the perforation against the flow direction also seems to result in higher reactive values at the lower frequencies. However, also a difference in frequency dependency is observed leading to a crossing of the reactance curves of both orientations. The frequency where both reactance curves cross seems to be lower for lower flow rates and higher inclination angles.

Based on the results presented in this paper, it is clear that flow rate, inclination angle and inclination orientation have a significant influence on the measured flow acoustic impedance of sub-millimeter perforations and it is hypothesized that the occurring effects can be attributed to the change in flow profile around the perforation opening. The specific physical nature and driving parameters hereof are, however, not fully clear and require further investigation. To this extend, the current test rig set-up will be further improved and different flow profiles will be studied.

\section{Acknowledgments}

The research of S. Vandemaele (fellowship no. 1S24920N) is funded by a grant from the Research Foundation Flanders (FWO). Also the Internal Funds KU Leuven are gratefully acknowledged for their support.

\section{References}

[1] Maa, D.-Y., "Microperforated panel wide-band absorber," Noise Control Engineering Journal, Vol. 29, 1987, pp. 77-84.

[2] Maa, D.-Y., “Theory and design of microperforated-panel sound-absorbing construction,” Scientia Sinica, Vol. 18, 1975, pp. $55-71$.

[3] Maa, D.-Y., "Potential of microperforated panel absorber," The Journal of the Acoustical Society of America, Vol. 104, No. 5, 1998, pp. 2861-2866. 
[4] Allam, S., and Åbom, M., "A new type of muffler based on microperforated tubes," Journal of Vibration and Acoustics, Vol. 133, No. 3, 2011, pp. $0310051-8$.

[5] Yu, X., Cheng, L., and You, X., "Hybrid silencers with micro-perforated panels and internal partitions," The Journal of the Acoustical Society of America, Vol. 137, No. 2, 2015, pp. 951-962.

[6] Allam, S., and Åbom, M., “Cooling fan noise control using micro-perforates,” DIVA, 2012, pp. 10434-10445.

[7] Elnady, T., "Modelling and characterization of perforates in lined ducts and mufflers (Paper III)," Ph.D. thesis, Royal Institute of Technology (KTH), 2004.

[8] Kooi, J., and Sarin, S., "An experimental study of the acoustic impedance of Helmholtz resonator arrays under a turbulent boundary layer," 7th AIAA Aeroacoustics Conference, 1981, pp. AIAA-81-1998.

[9] Vandemaele, S., Denayer, H., De Roeck, W., and Desmet, W., "Numerical modeling of the flow acoustic behavior of sub-millimeter orifices in 3D using linearized Navier-Stokes equations," 25th AIAA/CEAS Aeroacoustics Conference, 2019, p. 2628 .

[10] Ronneberger, D., "The acoustical impedance of holes in the wall of flow ducts," Journal of Sound and Vibration, Vol. 24, No. 1, 1972, pp. 133-150.

[11] Dean, P., "An in situ method of wall acoustic impedance measurement in flow ducts," Journal of Sound and Vibration, Vol. 34, No. 1, 1974, pp. 97-IN6.

[12] Kooijman, G., Hirschberg, A., and Golliard, J., "Acoustical response of orifices under grazing flow: Effect of boundary layer profile and edge geometry," Journal of Sound and Vibration, Vol. 315, No. 4, 2008, pp. 849-874.

[13] Rayleigh, L., Theory of sound II, MacMillan, New York, 1929. P. 327.

[14] Peat, K. S., Ih, J.-G., and Lee, S.-H., "The acoustic impedance of a circular orifice in grazing mean flow: Comparison with Theory," The Journal of the Acoustical Society of America, Vol. 114, No. 6, 2003, pp. 3076-3086.

[15] De Roeck, W., Baelmans, M., and Desmet, W., "Aerodynamic/acoustic splitting technique for aomputation aeroacoustics applications at low-Mach numbers," AIAA Journal, Vol. 46, No. 2, 2008, pp. 463-475.

[16] Dokumaci, E., "Sound transmission in narrow pipes with superimposed uniform mean flow and acoustic modelling of automobile catalytic converters," Journal of Sound and Vibration, Vol. 182, No. 5, 1995, pp. 799-808.

[17] Dokumaci, E., "A note on transmission of sound in a wide pipe with mean flow and viscothermal attenuation," Journal of Sound and Vibration, Vol. 208, No. 4, 1997, pp. 653-655.

[18] Tijdeman, H., "On the propagation of sound waves in cylindrical tubes," Journal of Sound and Vibration, Vol. 39, No. 1, 1975, pp. 1-33.

[19] Fujimori, T., Sato, S., and Miura, H., "An automated measurement system of complex sound pressure reflection coefficients," INTER-NOISE and NOISE-CON Congress and Conference Proceedings, Vol. 1984, Institute of Noise Control Engineering, 1984, pp. 1009-1014.

[20] Ingard, U., and Singhal, V. K., "Upstream and downstream sound radiation into a moving fluid," The Journal of the Acoustical Society of America, Vol. 54, No. 5, 1973, pp. 1343-1346.

[21] Sivian, L. J., "Acoustic impedance of small orifices," The Journal of the Acoustical Society of America, Vol. 7, No. 2, 1935, pp. 94-101.

[22] Ingard, U., and Ising, H., "Acoustic nonlinearity of an orifice," The Journal of the Acoustical Society of America, Vol. 42, No. 1, 1967, pp. 6-17.

[23] Denayer, H., De Roeck, W., and Desmet, W., "Iterative procedure for accurate plane wave decomposition in flow ducts," Proceedings of the 26th Conference on Noise and Vibration Engineering (ISMA2014), Vol. 26, 2014, pp. $279-288$.

[24] Melling, T. H., "The acoustic impendance of perforates at medium and high sound pressure levels," Journal of Sound and Vibration, Vol. 29, No. 1, 1973, pp. 1-65.

[25] Guess, A., "Calculation of perforated plate liner parameters from specified acoustic resistance and reactance," Journal of Sound and Vibration, Vol. 40, No. 1, 1975, pp. 119-137.

[26] Dickey, N., Selamet, A., and Ciray, M., "An experimental study of the impedance of perforated plates with grazing flow," The Journal of the Acoustical Society of America, Vol. 110, No. 5, 2001, pp. 2360-2370. 